\title{
Suppression of Oct4 by Germ Cell Nuclear Factor Restricts Pluripotency and Promotes Neural Stem Cell Development in the Early Neural Lineage
}

\author{
Wado Akamatsu, ${ }^{1,2}$ Brian DeVeale, ${ }^{1}$ Hideyuki Okano, ${ }^{2}$ Austin J Cooney, ${ }^{3}$ and Derek van der Kooy ${ }^{1}$ \\ ${ }^{1}$ Department of Molecular Genetics, University of Toronto, Toronto, Ontario, Canada M5S3E1, ${ }^{2}$ Department of Physiology, Keio University School of \\ Medicine, Tokyo 160-8582, Japan, and ${ }^{3}$ Department of Molecular and Cellular Biology, Baylor College of Medicine, Houston, Texas 77030
}

\begin{abstract}
The earliest murine neural stem cells are leukemia inhibitory factor (LIF)-dependent, primitive neural stem cells, which can be isolated from embryonic stem cells or early embryos. These primitive neural stem cells have the ability to differentiate to non-neural tissues and transition into FGF2-dependent, definitive neural stem cells between embryonic day 7.5 and 8.5 in vivo, accompanied by a decrease in non-neural competency. We found that Oct 4 is expressed in LIF-dependent primitive neural stem cells and suppressed in FGF-dependent definitive neural stem cells. In mice lacking germ cell nuclear factor (GCNF), a transcriptional repressor of Oct4, generation of definitive neural stem cells was dramatically suppressed, accompanied by a sustained expression of Oct4 in the early neuroectoderm. Knockdown of Oct4 in $G C N F^{-1-}$ neural stem cells rescued the $G C N F^{-1-}$ phenotype. Overexpession of Oct 4 blocked the differentiation of primitive to definitive neural stem cells, but did not induce the dedifferentiation of definitive to primitive neural stem cells. These results suggested that primitive neural stem cells develop into definitive neural stem cells by means of GCNF induced suppression of Oct4. The Oct4 promoter was methylated during the development from primitive neural stem cell to definitive neural stem cell, while these neural stem cells lose their pluripotency through a GCNF dependent mechanism. Thus, the suppression of Oct4 by GCNF is important for the transition from primitive to definitive neural stem cells and restriction of the non-neural competency in the early neural stem cell lineage.
\end{abstract}

Key words: stem cells; development; ectoderm; differentiation; growth factor; neural precursor

\section{Introduction}

During mouse development, the earliest neural stem cells (expressing neural tissue specific markers) can be isolated at E5.5 using clonal neurosphere cultures (Hitoshi et al., 2004). These primitive neural stem cells (pNSCs) are dependent on leukemia inhibitory factor (LIF) and are similar to the pNSCs from embryonic stem (ES) cells (Tropepe et al., 2001). Both ES and embryonic epiblast derived pNSCs transition clonally into FGFdependent definitive neural stem cells (dNSCs) (Tropepe et al., 2001; Hitoshi et al., 2004), which are similar to the dNSCs isolated from the later embryonic (Reynolds and Weiss, 1992) or adult (Morshead et al., 1994) mammalian nervous system. This conversion from pNSC to dNSC occurs between embryonic day 7.5 (E7.5) and E8.5 (Hitoshi et al., 2004). pNSCs are specified to neural tissue but have ability to contribute to non-neural lineages by making blastocyst chimeras (Tropepe et al., 2001), while

\footnotetext{
Received Sept. 22, 2008; revised Jan. 6, 2009; accepted Jan. 8, 2009.

This work was supported by a Canadian Institutes of Health Research Grant. W.A. was supported by a Huntington's Disease Society of America Research Fellowship, a Uehara Memorial Foundation Research Fellowship, and a Foreign Research Fellowship of the Japan Pediatric Society. B.D. was supported by a Vision Science Research Program Award. Thanks to Dr. Tomoyuki Inoue, Brenda Coles, and Susan Runciman for technical assistance and valuable comments, and Tania Alexson for helpful discussions.

Correspondence should be addressed to either of the following: Wado Akamatsu or Derek van der Kooy, Department of Molecular Genetics, University of Toronto, 160 College Street Room 1102, Toronto, Ontario, Canada M5S3E1.

E-mail:wado@sc.itc.keio.ac.jp; or derek.van.der.kooy@utoronto.ca.

D0I:10.1523/JNEUROSCI.4527-08.2009

Copyright $\odot 2009$ Society for Neuroscience $\quad 0270-6474 / 09 / 292113-12 \$ 15.00 / 0$
}

dNSCs do not (Tropepe et al., 2001; Seaberg et al., 2002; Karpowicz et al., 2007). However, the molecular mechanisms that induce a loss of non-neural competency in early NSCs remain unclear.

Oct4, a POU-family transcription factor, is required for the maintenance of pluripotency and the self-renewal of ES cells (Niwa et al., 2000; Orkin, 2005; Smith, 2005). Oct4 also is required for the pluripotency of the inner cell mass of the developing blastocyst (Nichols et al., 1998), as well as early lineage specification in vivo (Niwa et al., 2005). Oct4 expression gradually becomes restricted primarily to the germ cell lineage after gastrulation (Yeom et al., 1996). The expression of Oct4 in the developing early neuroectoderm is suppressed and restricted by an orphan nuclear receptor, germ cell nuclear factor (GCNF) (Fuhrmann et al., 2001). GCNF is a transcriptional repressor that downregulates target gene expression through binding to response elements (Hummelke and Cooney, 2001). GCNF is expressed in mouse embryos as early as E6.5, and continues to be expressed in the anterior neuroepithelium at E8.5. By E9.5, GCNF expression is markedly reduced in the forebrain and midbrain regions and is restricted in germ cells in the adult (Chung and Cooney, 2001; Lan et al., 2003; Chung et al., 2006). Oct4 is a direct target of GCNF and Oct4 expression inversely correlates with GCNF expression in developing somatic embryonic cells (Fuhrmann et al., 2001). Mouse embryos lacking GCNF die at E10.5 with cardiovascular failure, abnormal posterior development and insufficient closure of neural tube and impaired mid- 
brain development (Chung et al., 2001; Chung et al., 2006) accompanied with a sustained Oct4 expression in the developing neuroectoderm (Fuhrmann et al., 2001).

Possible functions of Oct4 after gastrulation in vivo, especially with respect to the neural lineage, have remained unexplored due to the subtle expression of Oct 4 in the neuroectoderm and the early growth arrest of Oct4 ${ }^{-/-}$embryo (Nichols et al., 1998). Given that $G C N F^{-1-}$ embryos show sustained expression on Oct4 in the embryonic neuroectoderm, we have focused on the neural lineage specific Oct4 suppression by GCNF to explore the role of Oct 4 in the early neural stem cell lineage. In this report, we show that GCNF induced suppression of Oct4 in the developing neuroectoderm promotes the transition from pNSC to dNSCs and also restricts the potential of these dNSCs to form non-neural cell types.

\section{Materials and Methods}

Animals and immunohistochemistry. GCNF deficient mice (Chung et al., 2001) on the C57BL/6 background were used in this study. Genotyping was performed by genomic PCR as described (Chung et al., 2001). Homozygous embryos were obtained by breeding heterozygous animals. Embryos were fixed in $4 \%$ paraformaldehyde for $6 \mathrm{~h}$ and then placed in $30 \%$ sucrose overnight and embedded in Tissue-tek mounting media. Twelve micrometer sections were used for immunostaining. Anti-Oct4 monoclonal antibody (BD Biosciences), anti-GFP monoclonal antibody (Invitrogen), anti-Nestin polyclonal antibody (a gift from Ron McKay, National Institute of Neurological Disorders and Stroke, Bethesda, MD), anti-GFP polyclonal antibody (Invitrogen), anti-human $\alpha$-1-fetoprotein (AFP) polyclonal antibody (Dako) and anti-Pax6 monoclonal antibody (DSHB) were used in dilutions of 1:500, 1:100, 1:500, 1:500, 1:500, and 1:500, respectively. Alexa 568 conjugated anti-mouse IgG antibody, Alexa 568 conjugated anti-rabbit IgG antibody, Alexa 488 conjugated antirabbit IgG antibody and Alexa 488 conjugated anti-mouse IgG antibody were used 1:1000, 1:1000, 1:500 and 1:500, respectively. Cell nuclei were counterstained with the nuclear dye DAPI or Hoechst 33258.

In situ hybridization. A $242 \mathrm{bp}$ probe amplified from full-length GCNF cDNA using two primers (supplemental Table S1, available at www. jneurosci.org as supplemental material) was subcloned into the pCR2.1 (Invitrogen) vector. SP6 and T7 RNA polymerase were used for synthesizing sense and antisense digoxigenin labeled probes. The probes were hybridized to $12 \mu \mathrm{m}$ cryostat sections. Detailed protocols will be provided upon request.

Primary cell culture. The isolation of neural stem cells from mice was performed as previously described (Hitoshi et al., 2004). The passaging procedures, differentiation assays, and immunohistochemical analyses have been described previously (Tropepe et al., 2001; Hitoshi et al., 2002).

Immunocytochemistry. Fixation and immunocytochemical analysis of cells was performed as described previously (Smukler et al., 2006).

$R T-P C R$ and quantitative RT-PCR. Total RNA was isolated using the RNeasy extraction kit mini (Qiagen) or Trizol reagent (Invitrogen), and cDNA was synthesized using random primers and MuMLV reverse transcriptase (Superscript III, Invitrogen) at $42 \mathrm{C}$ for $1 \mathrm{~h}$. Cybergreen qPCR mix (Applied Biosystems) was used for quantitative PCRs. Primer sequences and specific PCR conditions for Oct4, Nanog, GAPDH, GCNF, GATA-4, Brachyury-T, GFAP, Neurogenin- 2 and $\beta$-actin will be provided upon request. For quantitative PCR, the ABI 7000 real-time thermalcycler (Applied Biosystems) was used.

Immunoblotting. Anti-Oct4 monoclonal antibody (BD Biosciences) and anti- $\beta$-Actin monoclonal antibody (Sigma) were used in dilutions of $1: 500$ and 1:5000, respectively. HRP-conjugated anti-mouse IgG secondary antibody (Jackson) and ECL (Amersham) were used for the detection.

ES cell cultures. The $G C N F^{+/+}$and $G C N F^{-/-}$ES cells (Gu et al., 2005) were grown on mitotically inactive fibroblast feeder layers and maintained in DMEM plus 15\% FCS culture medium containing LIF (1000 $\mathrm{U} / \mathrm{ml}$ ) at low passage numbers $(6 \sim 11)$, as previously described (Tropepe et al., 2001). ES cells were trypsinized and plated in serum-free media at 10 cells $/ \mu \mathrm{l}$ in 24 well culture plates in the presence of LIF $(1000 \mathrm{U} / \mathrm{ml})$ or/and FGF2 $(10 \mathrm{ng} / \mathrm{ml})$ for $7 \mathrm{~d}$. Neurospheres (diameter, $>100 \mu \mathrm{m})$ were counted. To assess secondary spheres, each primary neurosphere was dissociated individually and plated in serum-free media with LIF and/or FGF for $7 \mathrm{~d}$. Differentiation of neuron and glia from neurosphere were described in a previous report (Tropepe et al., 2001). Short-term survival assays were performed as described (Smukler et al., 2006).

Generation of lentiviral vectors. Replication-defective, self-inactivating lentiviral vectors (Miyoshi et al., 1998; Tahara-Hanaoka et al., 2002) with EF $1 \alpha$ as an internal promoter were used. pCSII-EF (a gift from Dr. H. Miyoshi, RIKEN, Ibaraki, Japan) or pCSEIE (which contains an internal ribosome entry site (IRES)-EGFP) were modified and used for gene transfer into NSCs. For viruses expressing siRNA, the mouse U6 promoter was isolated from the mouse genome using primers $\mathrm{U} 6-5^{\prime}$ and U6-3' (supplemental Table S1, available at www.jneurosci.org as supplemental material) and replaced with the EF promoter of pCSII-EF. Then, a phosphoglycerate kinase (PGK) promoter-EGFP fluorescent reporter fragment was inserted into the vector. Oligonucleotides for siRNA were synthesized as described in (supplemental Table S1, available at www.jneurosci.org as supplemental material) and annealed to make double stranded. Then they were subcloned into the vector. A lentiviral vector which overexpresses Oct4 was generated by subcloning the Oct4 full-length cDNA into pCSEIE.

Bisulfite genomic sequencing. The genomic DNA from ES cells was extracted with a QIAGEN DNeasy kit. Purified genomic DNA was denatured and converted with an Epitect kit QIAGEN. The bisulfite-modified DNA was purified and used as a template for PCR (supplemental Table S1, available at www.jneurosci.org as supplemental material). The 900-bp PCR products were subcloned into the PCR2.1 cloning vector, and individual clones were randomly selected for DNA sequencing with M13 forward or reverse primers.

ChIP assay. Chromatin immunoprecipitation (ChIP) assays was performed as described (Gu et al., 2006).

Morula aggregation. Morula aggregation using 2 or $3 \mathrm{~d}$ small FGF-2dependent dNSC-derived neurosphere colonies from $\mathrm{GCNF}^{+/+}$or $G C N F^{-1-}$ ES cells was performed as described previously (Nagy et al., 2002), using Institute of Cancer Research (ICR) host morula.

Microscopy. Fluorescent images were visualized using a motorized inverted research microscope (IX81; Olympus) with $20 \times / 0.40$ and $40 \times /$ 0.60 objectives at room temperature and captured using Olympus MicroSuite Version 3.2 image analysis software (Soft Imaging System). Images were prepared using Photoshop CS3 (Adobe) software.

Statistics. All data are expressed as means $\pm \operatorname{SEM}(n>3)$. Comparisons were made using ANOVA or Student's $t$ tests. Significance was defined as two-tailed $p<0.05$.

\section{Results \\ Oct4 is progressively suppressed by germ cell nuclear factor in the early neural cell lineage}

We first analyzed the expression of Oct4 in ES cells, LIF dependent pNSC-derived neurospheres directly generated from single ES cells and FGF-dependent dNSC-derived neurospheres generated clonally from LIF dependent primary pNSC-derived neurospheres in vitro. By both RT-PCR (Fig. $1 A$ ) and immunoblotting (Fig. $1 B$ ), Oct4 was not detected in dNSC-derived neurospheres, but was detected in pNSC-derived neurospheres and ES cells. Oct4 and Nestin expression were examined in E8.5 neural tubes in vivo at the midbrain and spinal cord levels by immunostaining. Neither the E8.5 midbrain nor spinal cord contained any Oct4 positive cells (Fig. 1C). We further examined the expression of GCNF (Süsens et al., 1997; Gu et al., 2005). In situ hybridization revealed that GCNF mRNA was strongly expressed in the forebrain and midbrain at E8.5 and then dramatically diminished at E9.5 (Fig. 1D), suggesting that GCNF may suppress Oct4 expression in the neural tube until E8.5 before GCNF is downregulated by E9.5. To investigate whether Oct4 levels actually are affected in 
A

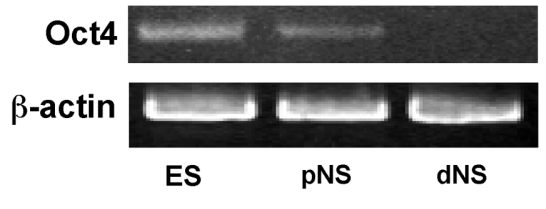

B

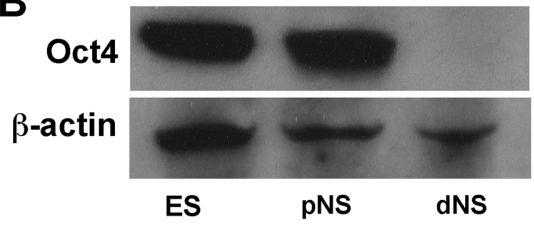

ES

D

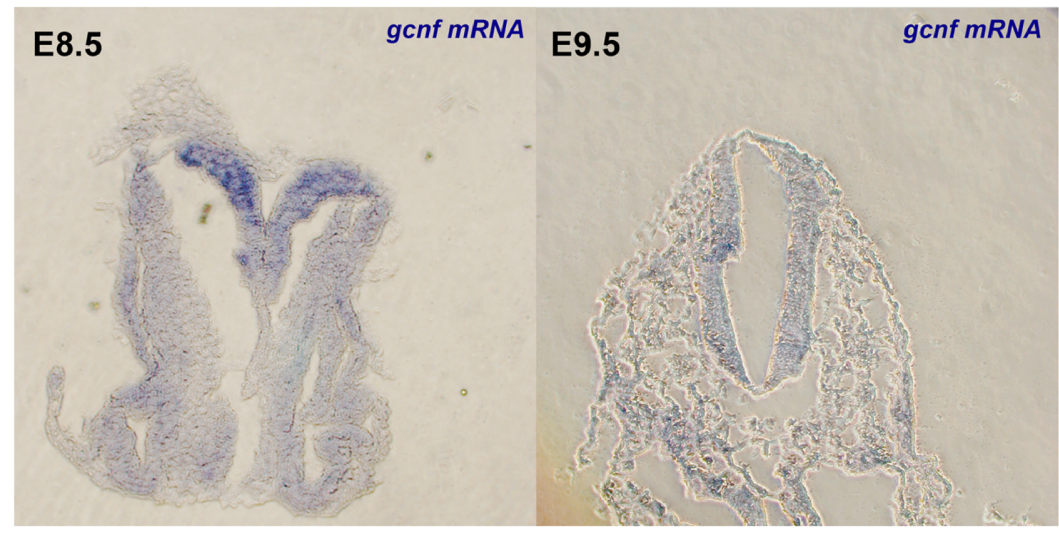

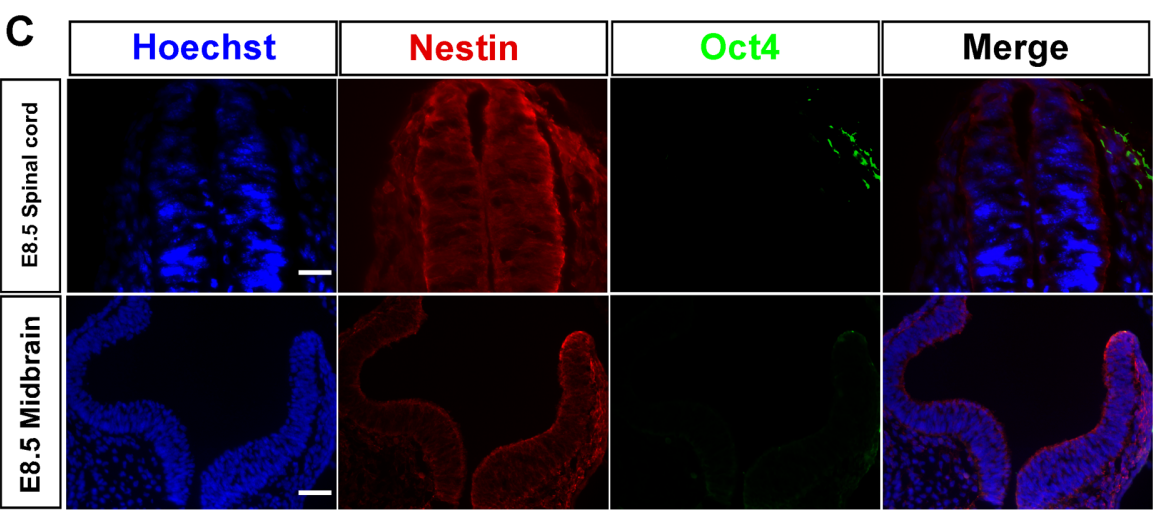

E8.5 neural tube

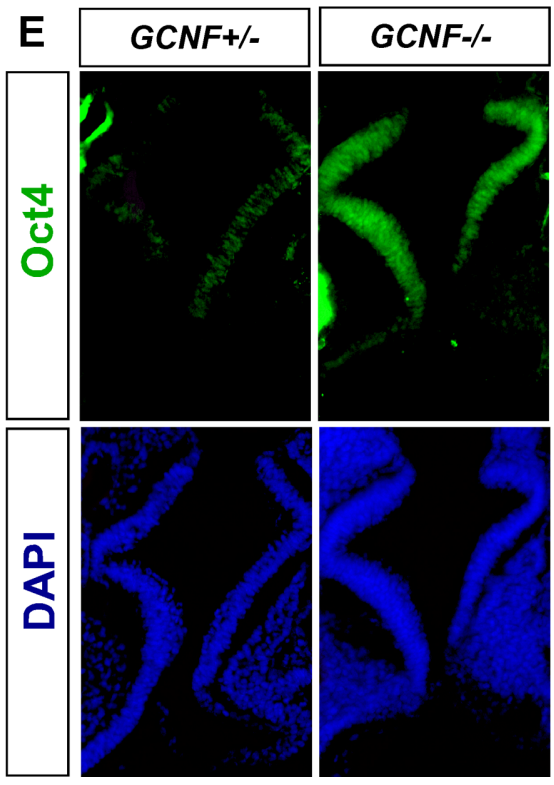

Figure 1. Oct4 is suppressed by germ cell nuclear factor (GCNF) in the early mouse neural tube. $A, B, 0 c t 4$ expression in ES cells, pNS- and dNSC-derived neurospheres generated from ES cells were analyzed by RT-PCR $(\boldsymbol{A})$ and immunoblotting $(\boldsymbol{B}) . \beta$-actin was used as an internal control. $\boldsymbol{C}$, Transverse sections of E8.5 mouse spinal cord (top) or midbrain (bottom) were double-immunostained using Nestin and $0 \mathrm{ct} 4$ antibodies. Scale bar, $250 \mu \mathrm{m}$. D, Expression of GCNF mRNA in E8.5 and E9.5 mouse neural tubes by in situ hybridization. $\boldsymbol{E}$, Transverse sections from the E8.5 mouse forebrain were immunostained by using 0ct4 antibody. pNS, pNSC-derived neurospheres; dNS, dNSC-derived neurospheres.

E8.5 neural tubes lacking GCNF, we examined Oct4 expression in E8.5 $\mathrm{GCNF}^{-1-}$ neural tubes at the forebrain level. Compared with their heterozygous controls, E8.5 $\mathrm{GCNF}^{-1-}$ embryos showed significantly increased amount of Oct 4 immunoreactivity throughout the neural tissue (Fig. $1 E$ ). These results suggest that GCNF suppresses Oct4 levels in the neural tube before E8.5, during the transition from pNSCs to dNSCs in vivo.

Loss of GCNF prevents the transition from LIF-dependent primitive neural stem cells to FGF-dependent definitive neural stem cells in E7.5-E8.5 mouse embryos

To test the effects of the increased Oct 4 levels in the developing $G_{C N F}^{-1-}$ neuroectoderm on neural stem cell development in vivo, we generated clonally derived neurospheres from early $\mathrm{GCNF}^{-1-}$ embryos. The numbers of primary pNSC-derived neurospheres from the neuroectoderm of E6.5 and E7.5 $\mathrm{GCNF}^{-1-}$ embryo were not significantly different from those of WT controls (Fig. 2 A). However, the numbers of primary pNSCderived neurospheres from the neuroectoderm of E8.5 $\mathrm{GCNF}^{-1-}$ embryos were significantly increased compared with wild-type controls (Fig. $2 \mathrm{~B}$ ). In contrast, the numbers of primary dNSCderived neurospheres from the neuroectoderm of E8.5 $\mathrm{GCNF}^{-1-}$ embryos were significantly decreased compared with WT controls (Fig. 2C).

Each single primary pNSC-derived neurospheres from $\mathrm{GCNF}^{-1-}$ and WT embryos was dissociated into single cells in separate wells to assess the pNSC versus dNSC nature of the secondary clonal neurospheres that formed. Only pNSCs derived from $G_{C N F}{ }^{-1-}$ embryos, and not from WT embryos, could be maintained as secondary pNSCs with passaging in the presence of LIF alone (Fig. 2D). These results suggest that NSCs present in the neuroectoderm of E8.5 $\mathrm{GCNF}^{-1-}$ embryos retain LIFdependent pNSC characteristics in vitro longer than those derived from WT embryos which quickly mature to dNSCs.

Neural stem cells derived from $\mathrm{GCNF}^{-1-}$ embryos retain high expression of Oct4 but remain specified to the neural lineage To investigate the characteristics of the NSCs present in the $\mathrm{GCNF}^{-1-}$ neural tube, we quantified the amount of Oct4 mRNA in the pNSC-derived neurospheres and dNSC-derived neurospheres from E8.5 WT and $G C N F^{-1-}$ embryos. In WT spheres, Oct4 is essentially absent in dNSC-derived neurospheres. In contrast, $\mathrm{GCNF}^{-1-}$ dNSC-derived neurospheres expressed 10-fold greater amounts of Oct 4 compared with WT dNSC-derived neu- 
rospheres (Fig. 2E). These data suggest that the GCNF ${ }^{-1-}$ dNSC-derived neurospheres (at least as defined by FGF dependence) exhibit some characteristics more similar to pNSC-derived neurospheres than to dNSC-derived neurospheres from WT embryos. GCNF mRNA was $3.39 \pm$ 0.20 -fold increased in clonal dNSCderived neurospheres derived from E7.5 WT embryos compared with clonal pNSC-derived neurospheres derived from E8.5 WT embryos by quantitative RT-PCR.

To assess whether pNSC-derived neurospheres and dNSC-derived neurospheres from $\mathrm{GCNF}^{-/-}$embryos remain specified to the neural lineage, GATA-4 (an endodermal marker) and Brachyury-T (a mesodermal marker) expression was examined by RT-PCR. Neither pNSC- nor dNSC-derived neurospheres from E8.5 $\mathrm{GCNF}^{-1-}$ embryos expressed these markers, nor did the similar spheres (defined by growth factor dependence) from WT embryos (Fig. 2F). Furthermore, the fates of $\mathrm{GCNF}^{-1-}$ NSC progeny were examined after differentiation. LIF-dependent pNSCderived neurospheres from E7.5 embryos were placed on coverslips and cultured with $1 \%$ serum to induce differentiation. Seventy-two hours later, clonal spheres from E7.5 $\mathrm{GCNF}^{-1-}$ embryos generated TuJ-1 ( $\beta$-III tubulin) positive neurons as well as spheres from WT embryos (supplemental Fig. S1, available at www.jneurosci. org as supplemental material), and the ratios of TuJ-1 positive neurons to total cells were similar between $G C N F^{-1-}$ and $\mathrm{GCNF}^{+/+}$neurospheres (Fig. 2G). Together, these results suggest that NSCs derived from E7.5 $\mathrm{GCNF}^{-1-}$ embryos remain fated to the neural lineage.

$\mathrm{GCNF}^{-/-}$-ES-derived neural stem cells retain pNSC character and display sustained expression of Oct4

The characteristics of pNSCs and dNSCs isolated from $\mathrm{GCNF}^{+/+}$ and $G_{C N F^{-1-}}$ embryos were compared with those of neural stem cells isolated from ES cell clones established from GCNF deficient mice ( $\mathrm{Gu}$ et al., 2005). Clonal neurospheres were generated from $\mathrm{GCNF}^{+/+}$and $\mathrm{GCNF}^{-/-}$ES cells, in a serum-free floating culture with various growth factors and/or inhibitors (LIF, FGF and SU5402). GCNF ${ }^{-1-}$ ES cells generated slightly but significantly increased numbers of neurospheres compared with $\mathrm{GCNF}^{+/+} \mathrm{ES}$ cells (Fig. 3A). SU5402, a FGF-receptor tyrosine kinase signaling inhibitor, blocks the endogenous FGF signaling that is necessary for the proliferation of clonal neurospheres produced by ES cell derived pNSCs (Tropepe et al., 2001). However, pNSCs derived from $\mathrm{GCNF}^{-/-}$ES cells, but not $\mathrm{GCNF}^{+/+}$ES cells, were still able to generate neurospheres even in the presence of SU5402 (Fig. $3 A)$. This suggests that the sustained Oct4 expression in $G C N F^{-1-}$ pNSCs makes them less dependent of endogenous FGF, as they were able to generate neurospheres in the presence of
SU5402. To assess whether the increased neurospheres produce progeny that are specified to the neural lineage, pNSC-derived neurospheres from $G C N F^{+/+}$and $G C N F^{-/-}$ES cells were differentiated by adding FBS and withdrawing LIF from the medium. The frequencies of the spheres which generate neurons were not significantly changed in $G C N F^{-1-}(94.4 \pm 3.2 \%)$ compared with WT spheres $(91.9 \pm 1.8 \%)\left(t_{(4)}=0.72 ; p>0.05\right)$. To ask whether $\mathrm{GCNF}^{-1-}$ primary, ES cell derived, pNSCs show a similar prolonged retention of the pNSC phenotype as those pNSCs derived from $G C N F^{-1-}$ embryos, primary, pNSC-derived spheres from ES cells were dissociated to produce clonal secondary spheres in the presence of either LIF alone or LIF and FGF. Only GCNF ${ }^{-1-}$ primary neurospheres (but not WT spheres) were able to generate secondary spheres in the absence of FGF $(+/+; 2.4 \pm 0.67$ spheres, $-/-; 0.1 \pm 0.1$ spheres, $\left.t_{(13)}=4.76 ; p<0.05\right)$, while the numbers of secondary spheres generated in media which contains FGF and LIF from single primary pNSC-derived neuro- 


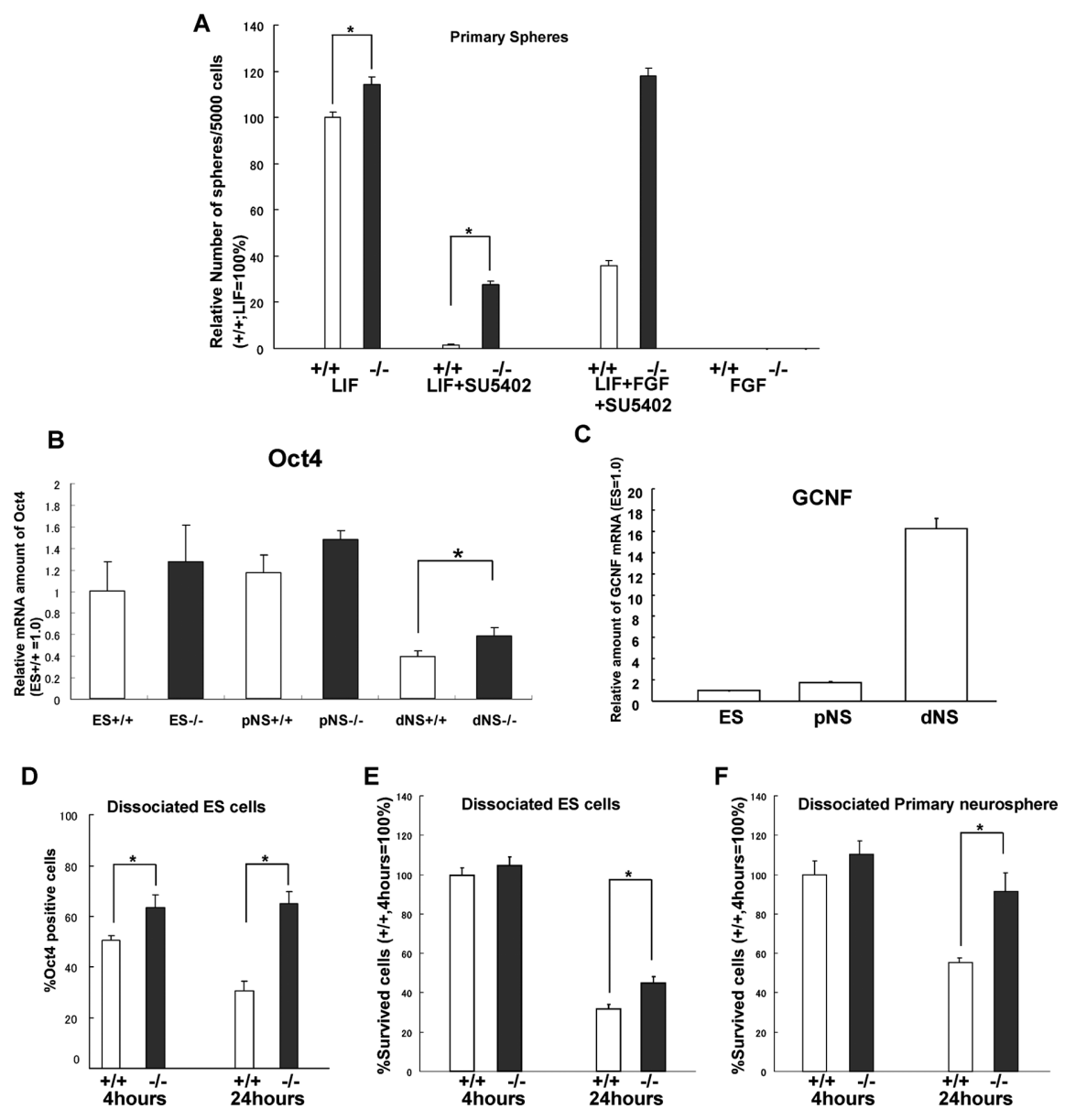

Figure 3. Characterization of primitive and definitive neural stem cell derived neurospheres from $\mathrm{GCNF}^{+/+}$and $\mathrm{GCNF}^{-/-} \mathrm{ES}$ cells. $A$, From GCNF ${ }^{+/+}$and GCNF ${ }^{-1-}$ ES cells, clonal pNSC-derived neurospheres were directly generated in serum-free floating culture with various growth factors. GCNF ${ }^{-1-}$ ES cells generated slightly but significantly increased numbers of neurospheres compared with $G C N F^{+/+}$ES cells $\left(t_{(14)}=3.60 ; p<0.05\right)$. GCNF ${ }^{-1-}$ ES cells were still able to generate neurospheres even in the presence of SU5402 $\left(t_{(14)}=18.9 ; p<0.05\right)$. B , Quantitative RT-PCR analysis of Oct4 expression in undifferentiated ES cells, pNSC-derived neurospheres and secondary dNSC-derived neurospheres from $\mathrm{GCNF}^{+/+}$and $\mathrm{GCNF}^{-/-}$ES cells. A two way ANOVA on genotypes and types of stem cells revealed a main effect of genotype $\left.F_{(1,12)}=6.316, p<0.05\right)$. C, Relative GCNF mRNA amounts in $G C N F^{+/+}$ES cells, pNSC-derived neurospheres and dNSC-derived neurospheres were quantified using real-time quantitative RT-PCR. D-F, GCNF ${ }^{+/+}$and GCNF ${ }^{-/-}$ES cells or pNSC-derived neurospheres colonies from ES cells were dissociated and plated in wells of 24-well dishes at a low-density (10 cells/ $\mu \mathrm{l} ; 500 \mu \mathrm{l} /$ well). Viable cells in the wells were stained by Hoechst 33342 and counted 4 and $24 \mathrm{~h}$ later. $0 \mathrm{ct} 4$ expression was examined using an anti-0ct4 antibody. D, Proportions of 0ct4 positive cells. The numbers of cells that express $0 \mathrm{ct} 4$ are significantly increased in the GCNF ${ }^{-1-}$ compared with $\mathrm{GCNF}^{+/+}$cells both 4 and $24 \mathrm{~h}$ after plating $\left(t_{(6)}=-2.41 ; p<0.05\right.$ in at $4 \mathrm{~h}, t_{(6)}=-5.48 ; p<0.05$ at $24 \mathrm{~h}$ ). $\boldsymbol{E}$, Relative numbers of viable cells from ES cells $\left(G C N F^{+/+}, 4 \mathrm{~h}=100 \%\right)$. $\boldsymbol{F}$, Relative numbers of viable cells from primary $\mathrm{pNSC}$-derived neurospheres $\left(\mathrm{GCNF}^{+/+}, 4 \mathrm{~h}=\right.$ $100 \%)$. In each of ES cells and dissociated primary pNSC-derived neurospheres, GCNF ${ }^{-1-}$ cells exhibit higher cell viability compared with $\mathrm{GCNF}^{+/+}$cells $24 \mathrm{~h}$ after plating $\left(t_{(6)}=-3.61 ; p<0.05\right.$ for ES cells $24 \mathrm{~h}, t_{(6)}=-3.61 ; p<0.05$ for pNSC $\left.24 \mathrm{~h}\right)$. pNS; pNSC-derived neurospheres, dNS; dNSC-derived neurospheres

spheres were not significantly different between $\mathrm{GCNF}^{+/+}$and $\mathrm{GCNF}^{-/-}$cells $(+/+; 1.1 \pm 0.17$ spheres, $-/-; 1.0 \pm 0.31$ spheres, $\left.t_{(13)}=0.29 ; p>0.05\right)$. However, $G C N F^{-/-}$secondary, pNSC-derived neurospheres generated more tertiary dNSCderived neurospheres than pNSC-derived neurospheres (LIF; $2 \pm$ 0.47 spheres, FGF;9.6 \pm 1.7 spheres, $\left.t_{(18)}=4.76 ; p<0.05\right)$, suggesting that $\mathrm{GCNF}^{-1-}$ NSCs eventually were able to become FGF-dependent even in the absence of GCNF.

Interestingly, pNS derived from both $\mathrm{GCNF}^{+/+}$and $G C N F^{-1-}$ ES cells expressed similar or slightly upregulated Oct4 mRNA levels compared with ES cells of each genotype (Fig. 3B). However, Sox 1 mRNA expression was increased in pNS, compared with ES cells of the same genotype (supplemental Fig. S2, available at www.jneurosci.org as supplemental material). Even
GFAP and Neurogenin-2 were detectable in both $\mathrm{GCNF}^{+/+}$and $\mathrm{GCNF}^{-1-}$ dNSCderived neurospheres, again suggesting a neural specification of the pNSCs (supplemental Fig. S3, available at www.jneurosci. org as supplemental material). Oct4 then was downregulated in both $\mathrm{GCNF}^{+/+}$and $\mathrm{GCNF}^{-1-}$ cells. However, GCNF${ }^{-1-}$ dNSC-derived neurospheres still maintained higher amounts of Oct 4 mRNA compared with $\mathrm{GCNF}^{+/+}$colonies (Fig. $3 B$ ). GCNF mRNA similarly was quantified in $\mathrm{GCNF}^{+/+}$ES cell colonies, pNSCderived neurospheres and dNSC-derived neurospheres (Fig. 3C). GCNF mRNA was increased greatly in clonal dNSC-derived neurospheres compared with ES cells and clonal pNSC-derived neurospheres. These results suggest that pNSCs directly derived from $G C N F^{-1-}$ ES cells have similar characteristics to pNSCs from $\mathrm{GCNF}^{-1-}$ embryos, in that they both are specified to the neural lineage and are delayed in the progression from pNSCs to dNSCs compared with the neural stem cells derived from WT embryos and ES cells.

The increased numbers of pNSCderived neurospheres from $\mathrm{GCNF}^{-/-}$ ES cells are due to increased cell survival of pNSCs and neural progenitors

To test whether the increased pNSCderived neurosphere formation from single $\mathrm{GCNF}^{-1-}$ compared with $\mathrm{GCNF}^{+/+}$ ES cells was due to increased neural specification or increased cell survival of $\mathrm{pN}$ SCs, a direct short-term (24 h) differentiation assay from ES cells was performed (Tropepe et al., 2001; Smukler et al., 2006). When dissociated single WT ES cells are cultured at low-density in the absence of serum and growth factors, the majority of cells begin neural specification and express Nestin $4 \mathrm{~h}$ later (before any cell loss is seen in these cultures), but by $24 \mathrm{~h}, 70 \%$ of the cells die and the majority of the remaining cells are neural (Tropepe et al., 2001; Smukler et al., 2006). Placing $G C N F^{+/+}$ES cells in these minimal media conditions resulted in a significant decrease in the numbers of Oct 4 positive cells between 4 and $24 \mathrm{~h}$, however no such decrease in the numbers of Oct4 positive cells was seen over the same period in $G_{C N F}{ }^{-1-}$ ES cells (Fig. 3D). Increased numbers of viable cells were observed in $\mathrm{GCNF}^{-1-}$ compared with $\mathrm{GCNF}^{+/+}$cells at $24 \mathrm{~h}$ after the placement both in minimal media conditions [neurosphere conditions with LIF (Fig. 3E)] (supplemental Fig. S4, available at www.jneurosci.org as supplemental material). We also examined Sox 1 expression in these cells. More than $95 \%$ of both $\mathrm{GCNF}^{+/+}$and $\mathrm{GCNF}^{-/-}$cells expressed Sox1 at $4 \mathrm{~h}$ after plating (supplemental Fig. S5, available at www.jneurosci.org as supplemental material). Cells from dissociated, pNSC-derived neurospheres, which consist mostly of neural progenitor cells, were examined similarly in short term, minimal culture media, 
survival assays. The viability of neural progenitor cells from $\mathrm{GCNF}^{-/-}$compared with $G C N F^{+/+}$pNSC was also increased between 4 and $24 \mathrm{~h}$ (Fig. $3 F$ ). We also examined Sox 1 and Oct 4 expression in single cells exposed to neurosphere conditions (with LIF). Most of the surviving cells in neurosphere conditions also expressed Sox1, but an increased number of Oct 4 positive cells was observed compared with the minimal conditions (supplemental Fig. S6, available at www.jneurosci.org as supplemental material). These results suggests that the increased numbers of pNSCs from $\mathrm{GCNF}^{-/-}$compared with $\mathrm{GCNF}^{+/+}$ ES cells were due to an increased cell survival of $\mathrm{GCNF}^{-1-}$ pNSCs rather than an increased fate change from ES cells to $\mathrm{pN}$ SCs. Indeed, more $\mathrm{GCNF}^{-1-}$ than $\mathrm{GCNF}^{+/+}$primitive neural cells maintain Oct4 expression.

\section{Suppression of Oct4 but not Nanog rescues the impaired neural stem cell development in $\mathrm{GCNF}^{-1-}$ neural stem cells}

Sustained Oct4 is correlated with the $G C N F^{-1-}$ phenotypes in pNSCs and dNSCs, but is the sustained Oct4 functionally responsible for these phenotypes? Nanog has been identified as another target gene which is suppressed by GCNF protein ( $\mathrm{Gu}$ et al., 2005). In mouse embryos, Nanog expression is restricted to the germ cell linage after implantation and is quickly downregulated during the neural differentiation of ES cells induced by retinoic acid (Chambers et al., 2003). However, Nanog mRNA was observed in clonal dNSC-derived neurospheres from WT ES cells by RT-PCR (supplemental Fig. S7, available at www.jneurosci.org as supplemental material). To test whether potential changes in either or both of Nanog and Oct4 were responsible for the $G C N F^{-1-}$ neural stem cell phenotypes, lentiviruses, which express siRNA for Oct 4 and various controls, were used (Fig. 4A). Primary pNSC-derived spheres from $\mathrm{GCNF}^{+/+}$and $\mathrm{GCNF}^{-/-}$ES cells were placed in 96 wells and dissociated mechanically to allow secondary clonal colonies to form in serum-free media containing LIF or FGF. Immediately after dissociation of the primary colonies, the cells were infected with single lentiviruses $(\mathrm{MOI}=10)$ and cultured for $7 \mathrm{~d}$ to assess secondary clonal colony formation by GFP (driven by pGK promoter) positive pNSCs (Fig. $4 B, C$ ).

In the absence of lentiviral transfection, single primary pNSCderived neurospheres were able to generate secondary pNSCderived neurospheres, but few dNSC-derived neurospheres compared with $G C N F^{+/+}$cells (Fig. $4 D$ ). This $G C N F^{-/-}$phenotype was not changed by the infection of the control GFP virus or by nanog siRNA. However, when $G C N F^{-1-}$ pNSCs were infected

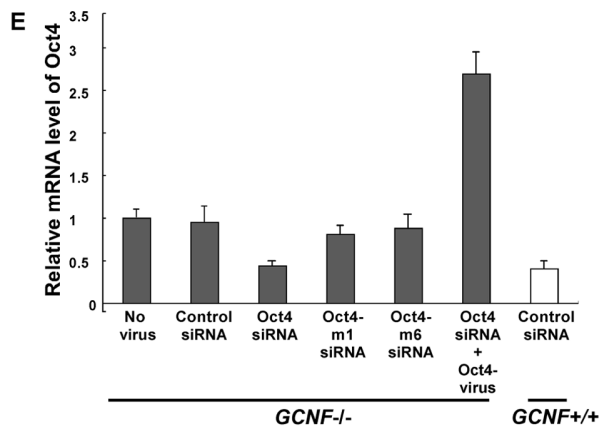

F

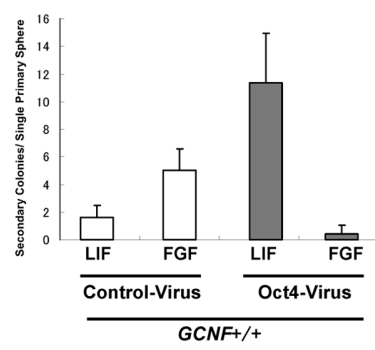

H

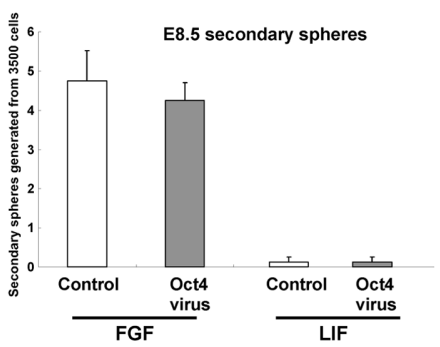

Figure 4. Suppression of $0 \mathrm{ct} 4$ rescues impaired neural stem cell development in $\mathrm{GCNF}^{-1-}$ neural stem cells but the transition from primitive to definitive neural stem cells is not reversible by 0ct4 overexpression. $A$, Structure of siRNA expressing lentiviral vectors. Dissociated primary pNSC-derived neurospheres from ES cells were cultured and infected with these lentiviruses in 96well GFP fluorescence throughout the spheres, indicating infection of the cell of origin $(\boldsymbol{B} ; \boldsymbol{C}$, bright field). $\boldsymbol{D}$, Mechanically dissociated cells from single primary pNSC-derived neurospheres were immediately infected by lentiviral vectors which express GFP/GFPA (control), GFP/Nanog-siRNA, GFP/0ct4 siRNA, GFP/0ct4-m1 siRNA (one mutation in 0ct4-siRNA) and GFP/0ct4-m6 (6 mutations in 0ct4-siRNA), and cultured in serum-free medium with LIF or FGF. Double infections of 0ct4-siRNA and 0ct4erived neurospheres (with/without lentiviral vectors) from $\mathrm{GCNF}^{-1-}$ ES or WT cells. 0ct4 was suppressed to $43 \%$ of that seen in control GCNF ${ }^{-1-}$ spheres. $\boldsymbol{F}$, The numbers of secondary colonies from dissociated single primary pNSC-derived neurospheres ES cells infected with control (GFP) or 0ct4 lentivirus. Growth factors were added as indicated. Oct4 overexpression creased the numbers of secondary colonies seen with LIF but decreased the numbers seen with FGF. G, The numbers of primary from dissociated forebrain germinal zone tissue from WT E14.5 CD1 embryos infected with control (GFP) or 0ct4 lentivius. Growth factors were added as indicated. No LIF-dependent colonies were observed. $\boldsymbol{H}$, The numbers of secondary colonies from dissociated primary dNSC-derived neurospheres from WT E8.5 CD1 embryos infected with control (GFP) or 0ct4 lentivirus. Growth factors were added as indicated.

with the lentivirus, which bore the siRNA for Oct4, then increased numbers of dNSC-derived neurospheres and decreased number of pNSC-derived neurospheres were observed compared with as well as pNSCs transfected with the control GFP siRNA lentivirus (Fig. 4D). Furthermore, Oct4-siRNA viruses harboring mutations (Oct4-m6, and Oct4-m1) did not induce any changes in the number of pNSC-derived neurospheres and dNSC-derived neurosphere colonies. To evaluate siRNA specificity, we did double infections of both the Oct4-siRNA virus and an Oct4overexpressing virus. With this double infection, the Oct4overexpressing virus was able to reverse the phenotype induced by Oct4-siRNA. However, the number of the FGF-dependent 
colonies after double infection was not smaller than that of the control-siRNA alone, suggesting that excessive amount of Oct4 cannot prevent dNSC generation completely. Oct4 and Nanog siRNA specifically suppressed the expression of their target genes to $43 \%$ (Fig. $4 E$ ) and $16 \%$ (supplemental Fig. S8, available at www.jneurosci.org as supplemental material), respectively, compared with control GFP siRNA in $\mathrm{GCNF}^{-/-}$secondary pNSCderived neurospheres. Oct4 protein expression was suppressed by Oct4 siRNA-lentivirus as well (supplemental Fig. S9, available at www.jneurosci.org as supplemental material). Thus, Oct4 knockdown is sufficient to revert the $G C N F^{-1-}$ phenotype in NSC development. We conclude that the sustained Oct4 expression in $G C N F^{-1-}$ NSCs caused the impaired transition from pNSCs to dNSCs.

\section{The transition from primitive to definitive neural stem cell is not reversible by Oct4 overexpression}

To determine whether direct Oct4 overexpression in pNSCs and dNSCs can affect the transitions between these two NSC types, dissociated primary pNS from WT ES cells were infected with a lentivirus expressing Oct4/GFP and then cultured for $7 \mathrm{~d}$ to allow clonal secondary neurosphere colonies to form. Oct4-overexpressing cells generated tenfold greater numbers of LIF-dependent, pNSC secondary neurospheres compared with control GFP only expressing cells. Furthermore, the number of Oct 4 overexpressing, secondary dNSCderived neurospheres was significantly decreased compared with control GFP only expressing cells (Fig. $4 F$ ). Quantitative RT-PCR revealed that the Oct4 overexpression vector produced 1.2 and 1.8fold increases in Oct4 expression in pNSC and dNSC neurospheres, respectively (supplemental Fig. S10, available at www.jneurosci.org as supplemental material). However, further passage of these Oct4overexpressing pNSC suggested that Oct4-overexpression was not sufficient to block completely the differentiation from pNSC to dNSC (supplemental Fig. S11, available at www.jneurosci.org as supplemental material). These results suggest that Oct4 overexpression induces a $G C N F^{-1-}$ phenotype in WT-ES-derived pNSCs. This also supports the interpretations suggested above: that the $G C N F^{-l-}$ phenotype in NSCs is due to increased Oct4 expression.

To determine whether Oct 4 overexpression can dedifferentiate brain dNSC-derived neurospheres, we overexpressed Oct 4 in primary dNSCs from the E14.5 mouse forebrain germinal zone. Primary, clonal neurosphere colonies were counted after $7 \mathrm{~d}$ of culture in either FGF or LIF. No pNSC-derived neurospheres were observed even if Oct4 was overexpressed (Fig. 4G). The numbers of dNSC-derived neurospheres were not significantly different between control and Oct4-overexpressing E14.5 NSCs. Furthermore, using dissociated dNSC-derived neurospheres derived from E8.5 WT embryos (presumably, most NSC were relatively early dNSCs at the start of the infection), only a very few pNSC-derived neurospheres were observed after $7 \mathrm{~d}$ culture in either the control and Oct 4 overexpression groups (Fig. $4 H$ ). The much larger numbers of dNSC-derived neurospheres were not significantly different between the control and Oct4-overexpression groups (Fig. $4 H$ ). These data suggest that Oct4 overexpression in WT pNSCs prevents their differentiation into dNSCs, but once $\mathrm{pN}$ SCs have transitioned into dNSCs they cannot be dedifferentiated into pNSCs again, even with Oct4 overexpression.

\section{GCNF induces de novo methylation of the Oct4 promoter during the transition from primitive to definitive neural stem cells}

GCNF initiates not only the repression but also epigenetic modification of the Oct4 gene during ES cell differentiation by recruit- ing MBD proteins (Gu et al., 2006). To evaluate the DNA methylation profile of the Oct4 promoter region during early neural stem cell differentiation, the methylation status of $16 \mathrm{CpG}$ sites in the Oct 4 gene (between -469 and the translation start site) was assessed at various stages during the development from ES cells to pNSC-derived neurospheres and to dNSC-derived neurospheres (by bisulfate treatment, PCR, and DNA sequencing) (Fig. $5 A$ ). In both $\mathrm{GCNF}^{+/+}$and $\mathrm{GCNF}^{-/-}$cells, the $\mathrm{CpG}$ sites remained mostly unmethylated in ES cells, primary pNSC-derived neurospheres, and in secondary and tertiary spheres grown in both FGF and LIF. However, in GCNF ${ }^{+/+}$dNSC-derived neurospheres (i.e., those grown only in FGF), methylated CpG sites in the Oct4 promoter region were significantly increased compared with $\mathrm{GCNF}^{+/+}$pNSC-derived neurospheres or to $\mathrm{GCNF}^{-/-}$dNSCderived neurospheres (Fig. $5 B$ ). We further analyzed dNSCderived neurospheres from E8.5, E14.5 mouse embryos and adult mouse brains. Oct $4 \mathrm{CpG}$ sites were methylated in dNSC-derived neurospheres from animals as well as in dNSC-derived neurospheres from ES cells (Fig. 5C). The direct binding of GCNF to the Oct4 promoter in WT ES cells was analyzed by ChIP assays. GCNF binding to the Oct4 promoter was enriched in dNSCderived neurospheres (Fig. 5D). These data suggest that de novo methylation of the Oct4 promoter is induced by GCNF during the transition from LIF-dependent, pNSCs to FGF-dependent, dNSCs in vitro and in vivo. Interestingly, the simple presence of FGF is not sufficient to promote this methylation even in the third passage neurospheres (Fig. 5A, B) if the pNSCs are grown in both LIF and FGF, suggesting that the loss of LIF may be more important than the presence of FGF for this NSC transition, at least in terms of methylation.

\section{$\mathrm{GCNF}^{-/-}$-ES-derived FGF-dependent definitive neural stem} cells generate chimeric early mouse embryos

Given that GCNF-dependent de novo methylation of Oct4 promoter is induced by removal of LIF, we examined plasticity of dNSC-derived neurospheres derived from $\mathrm{GCNF}^{+/+}$and $G C N F^{-1-}$ ES cells. Single cells from dissociated primary spheres were infected with a GFP expressing lentivirus to make clonal, wholly green fluorescent secondary neurospheres in the presence of both LIF and FGF. Then, single cells from dissociated secondary spheres were plated to make clonal tertiary colonies in the presence of FGF alone. Two or three days after, small fluorescent dNSC-derived neurospheres were cultured with E2.5 morula to make aggregation chimeras (Fig. 6A).

One day later, embryos were analyzed to ask whether fluorescent donor cells were integrated (Fig. $6 B, D$ ) or not integrated (Fig. $6 E-G$ ) into the inner cell mass of blastocysts. GCNF ${ }^{-/-}$ dNSC-derived colonies cultured with FGF alone for either 2 or $3 \mathrm{~d}$ (Fig. $6 \mathrm{H}$ ) showed an increased frequency of integration into host embryos compared with wild-type dNSC-derived colonies. During the 2 to $3 \mathrm{~d}$ period after LIF removal, $G C N F^{+/+}$dNSCs showed a reduced integration into in the inner cell mass of embryos from $33 \%$ to $5 \%$. In contrast, $\mathrm{GCNF}^{-1-}$ cells were able to integrate into the inner cell mass of $42 \%$ of embryos even $3 \mathrm{~d}$ after LIF removal.

$\mathrm{GCNF}^{-/-}$-ES-derived FGF-dependent definitive neural stem cells can contribute to non-neural tissue

Then, we implanted the blastocysts with successful aggregation of dNSCs into host pseudopregnant animals. Due to the limited number of blastocysts that integrate WT dNSCs (5\% using dNSCs 3 d after LIF removal)(Seaberg et al., 2002), we recovered only 5 embryos at E9.0, and only one of these (20\%) was chi- 
A

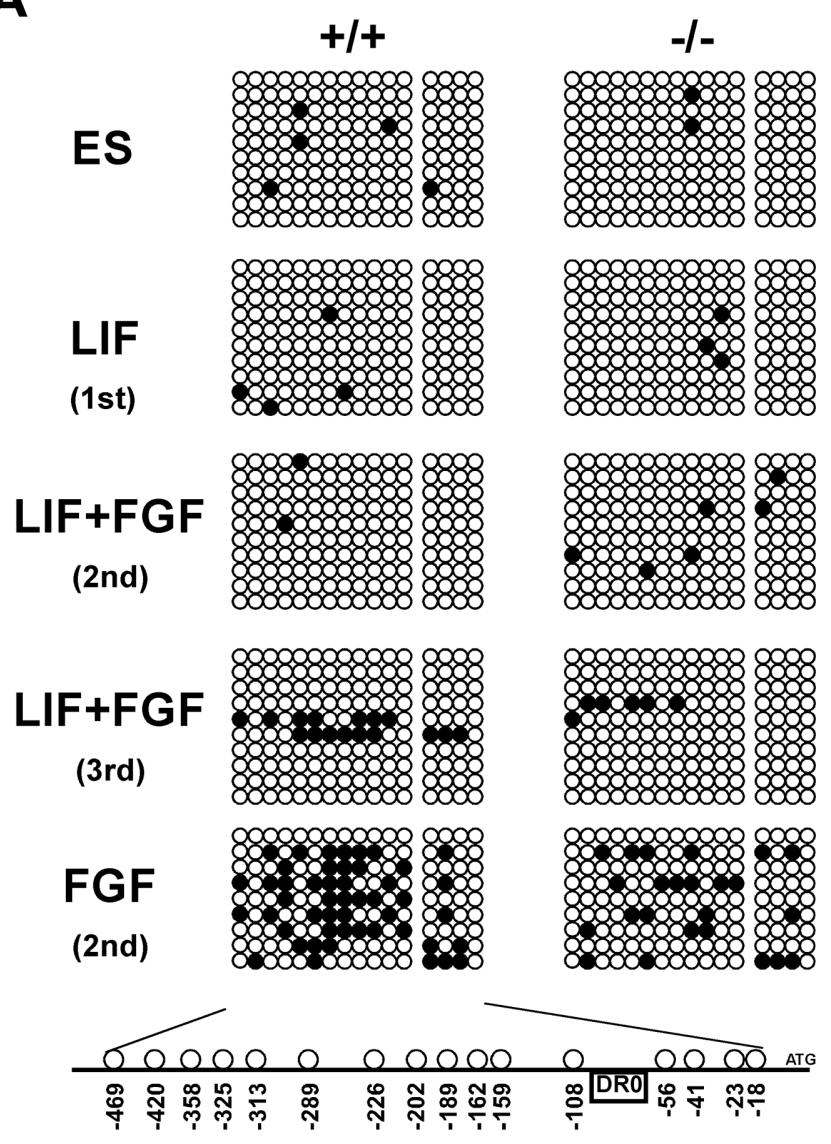

B

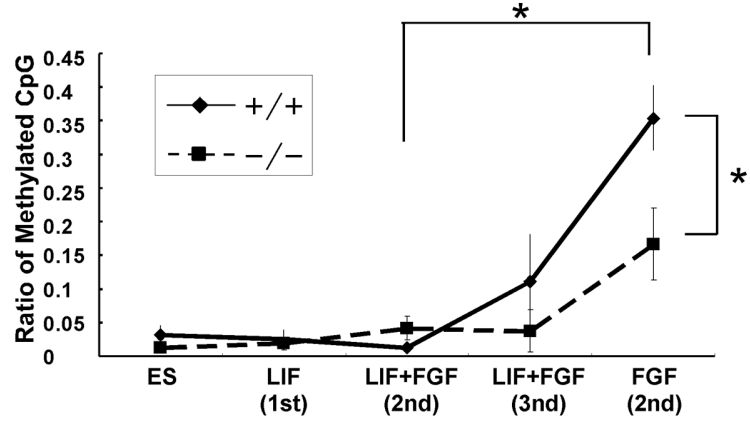

C

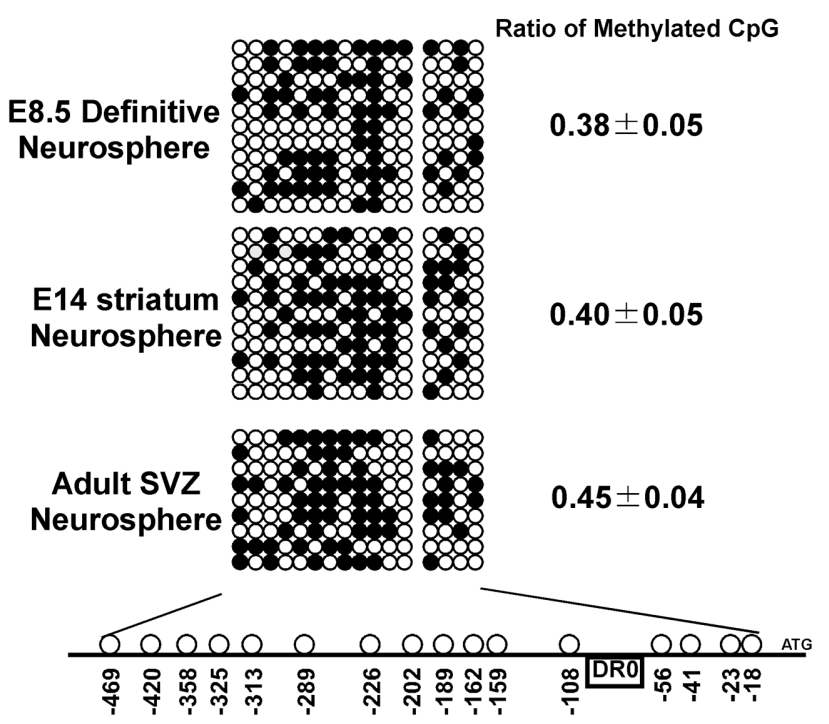

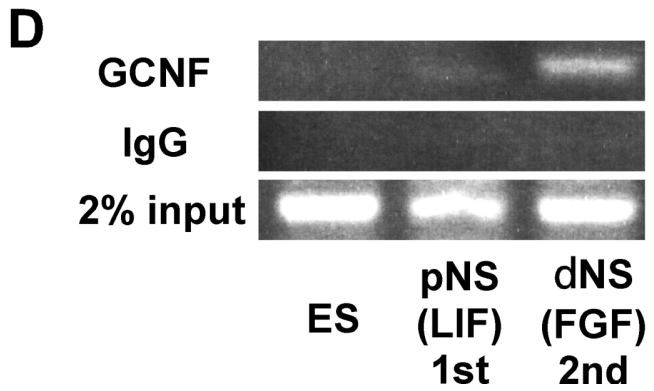

Figure 5. GCNF induces de novo methylation of the $0 \mathrm{ct} 4$ promoter during the transition from primitive to definitive neural stem cells. $A$, The DNA methylation profile of $16 \mathrm{CpG}$ sites located in the 0ct4 proximal promoter from -469 to the ATG start codon in ES cells, primary pNSC-derived neurospheres, secondary or tertiary LIF- and FGF-dependent spheres and secondary dNSC-derived neurospheres from $\mathrm{GCNF}^{+/+}$and $\mathrm{GCNF}^{-/-}$ES cells. The open circles represent unmethylated $\mathrm{CpG}$ dinucleotides, and the black circles represent methylated $\mathrm{CpG}$ sites. $\boldsymbol{B}$, Ratio of methylated to unmethylated $\mathrm{CpG}$ sites at $16 \mathrm{CpGs}$ in the 0ct4 proximal promoter. Methylation in GCNF ${ }^{+/+}$dNSC-derived neurospheres (FGF-second) is significantly increased compared with LIF + FGF neurospheres from GCNF ${ }^{+/+}$ES cells $\left(t_{(16)}=-2.69 ; p<0.05\right)$ or dNSC-derived neurospheres from $G_{C N F}{ }^{-1-}$ cells $\left(t_{(15)}=3.53 ; p<0.05\right)$. C, DNA methylation profile in the 0 ct4 proximal promoter in dNSC-derived neurospheres from WT E8.5, E14 and adult brain. Ratios indicate average ratios of methylated to unmethylated sites at the 16 CpG sites \pm SEM. D, Binding of GCNF to the 0 ct4 promoter in ES cells, pNSC-derived neurospheres and dNSC-derived neurospheres was detected by ChIP assays. Normal rabbit lgG was used as a control antibody.

meric, showing WT dNSC contributions to both neural and non neural tissue. However, four embryos were recovered from 20 implanted blastocysts which integrated $\mathrm{GCNF}^{-1-}$ dNSCs and all 4 chimeric embryos showed green fluorescent dNSC contribution to neural and non neural tissues at E9.0 (Fig. 7A,B). The frequency of chimeric embryo generated by $\mathrm{GCNF}^{-1-} \mathrm{dNSCs}$ was significantly higher than by $\mathrm{GCNF}^{+/+} \mathrm{dNSCs}\left(\mathrm{X}^{2}=0.016\right.$, $\chi^{2}$ test). GFP-positive donor cells were found in the heart wall
(Fig. 7C-E), endoderm with $\alpha$ FP expression (Fig. 7F-I) and neural tube with Pax6 expression (Fig. 7J-M). The high frequency of chimeric contribution in the recovered embryos with $\mathrm{GCNF}^{-1-}$ dNSCs suggest that GCNF $^{-1-}$ dNSCs have the ability to contribute to neural and non-neural tissue in vivo (as do WT pNSCs which contribute to $92 \%$ of E9.0 chimeras (Seaberg et al., 2002)), and that the loss of pluripotency induced by LIF removal in early neural stem cells is GCNF-dependent. 
A
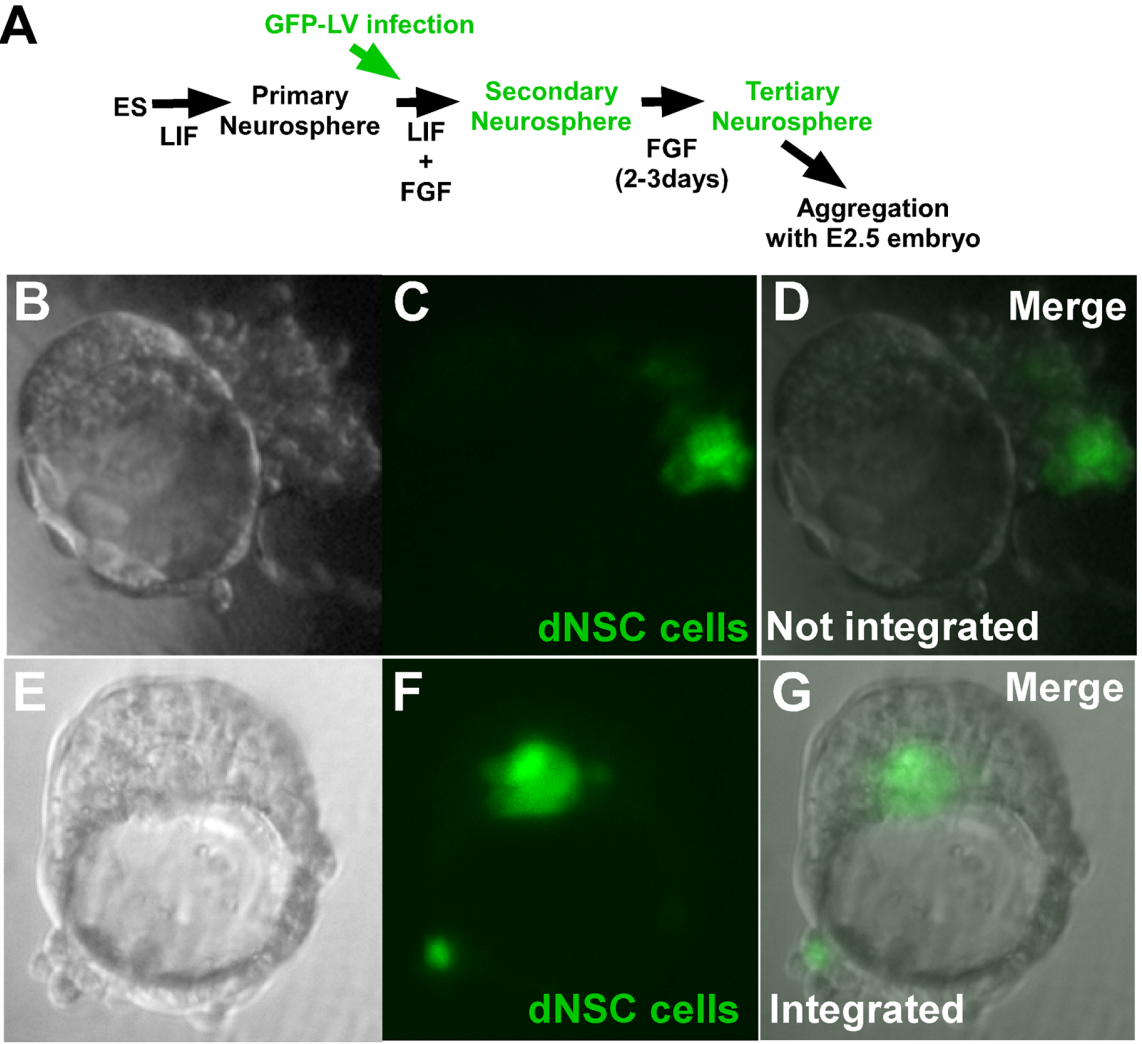

Not in

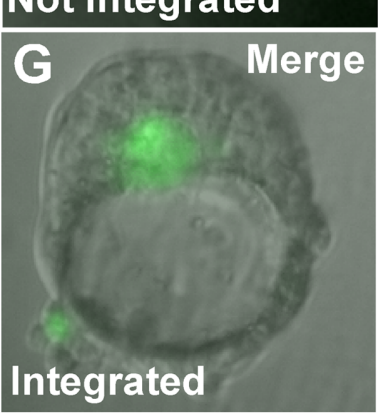

H

$(\%)$

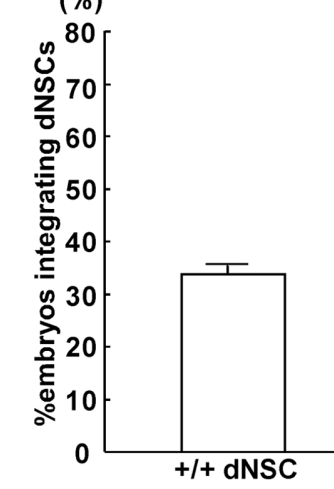

No. of embryos

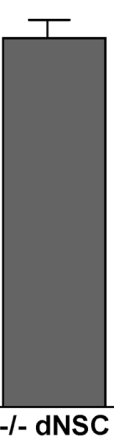

72
Integrated

\section{2 day tertiary dNSC}

Figure 6. $\mathrm{GCNF}^{-1-} \mathrm{dNSCS}$ generate chimeras with early mouse embryos. $A$, Strategy of the experiment. Dissociated primary neurospheres derived from $\mathrm{GCNF}^{+/+}$or ${ }^{-1-}$ ES cells were infected with a lentivirus that express GFP. Clonal secondary neurosphere cultured with LIF and FGF were GFP fluorescent. Dissociated secondary spheres were cultured with FGF and without LIF to make small tertiary neurospheres for 2 or $3 \mathrm{~d}$. Then, these neurospheres were cocultured with E2.5 host morula and cultured overnight to test aggregation. $\boldsymbol{B}-\boldsymbol{G}$, Representative pictures of aggregated embryos, which did not integrate $(\boldsymbol{B}-\boldsymbol{D})$ or did integrate $(\boldsymbol{E}-\boldsymbol{G})$ dNSCs into the inner cell mass of blastocysts after overnight incubation. dNSCs derived from ES cells were green fluorescent. $\boldsymbol{H}$, The percentages of embryos with integrated dNSCS. Left two bars show the data with $\mathrm{GCNF}^{+/+}$or ${ }^{-1-}$ tertiary neurospheres cultured for $2 \mathrm{~d}$ with FGF and without $\mathrm{LIF}\left(t_{(2)}=19.9 ; p<0.05\right)$. Right two bars show the data with similar tertiary neurospheres cultured for $3 \mathrm{~d}$ with FGF and without $\operatorname{LIF}\left(t_{(2)}=5.72 ; p<0.05\right)$. Both 2 and $3 \mathrm{~d} \mathrm{GCNF}{ }^{-1-}$ spheres showed an increased frequency of integration into host embryos compared with wild-type dNSC-derived colonies.

\section{Discussion}

The roles of Oct4 in developing embryonic somatic cells as they lose pluripotency have remained unknown due to the early lethality of Oct4 deficient animals. However, the present results suggest that Oct 4 acts as a suppressor of development in the early neural stem cell lineage. Both transcriptional suppression and epigenetic modification of the Oct 4 gene by GCNF occur during the transition from pNSC to dNSC, and they are necessary for this transition. Five pieces of evidence support this notion that GCNF suppression of Oct4 is necessary for the developmental switch from pNSCs to dNSCs in vivo and in vitro. First, GCNF expression precedes the suppression of Oct4 in the E7.5 developing neuroectoderm. Then by E8.5 Oct4 is suppressed, accompanied by increasing GCNF expression in vivo. Second, overexpression of Oct4 in pNSCs delays the transition from pNSCs into dNSCs in vitro. Third, the absence of GCNF in the early NSCs delays the transition from pNSC to dNSC in vivo and in neural stem cells derived from ES cells in vitro by increasing Oct4 level in dNSC similar to that of WT pNSC. Fourth, knockdown of Oct4 in GCNF ${ }^{-1-}$ pNSCs by specific siRNA rescues the impaired transition into dNSCs in vitro. Fifth, the epigenetic modification of the Oct 4 promoter allows the transition to dNSCs to be maintained even after GCNF is no longer expressed in mature dNSCs.

The present data show a clear asymmetry in the transition between clonal pNSCs and clonal dNSCs. pNSCs appear before and give rise to dNSCs both during in vivo embryonic development and in vitro when these cells are derived from ES cells. $\mathrm{GCNF}^{-1-}$ pNSCs derived from both mouse embryos and ES cells show delayed transitions into dNSCs. Oct4overexpressing pNSC from WT ES cells show a similar delayed transition for $\mathrm{pN}$ SCs to dNSCs. While the siRNA induced knockdown of the increased Oct4 in $\mathrm{GCNF}^{-/-}$pNSCs accelerates the transition into dNSCs, Oct4 overexpression in dNSC did not permit the generation of pNSCs from dNSCs. Furthermore, the gene silencing by methylation of the Oct4 promoter, which occurs during the transition from pNSC to dNSC, suggests a mechanism underlying the irreversibility of the transition from pNSC to dNSC. Neither loss of GCNF or overexpression of Oct4 (supplemental Fig. S11, available at www.jneurosci.org as supplemental material) completely prevents the eventual transition of pNSC into dNSC, perhaps due to the upregulation of another Oct4 suppressor, COUP-TF1 (supplemental Fig. S12A, available at www.jneurosci.org as supplemental material) or the epigenetic turn off of other pluripotency genes. GCNF also must silence other pluripotency genes as well as Oct4, because otherwise overexpression of Oct4 alone should have allowed the reversion from dNSC to pNSC. Recently, it has been reported that four defined factors including Oct4 are required for induction of pluripotent stem cells from mouse embryonic and adult fibroblast cells (Takahashi and Yamanaka, 2006), suggesting that synergis- 

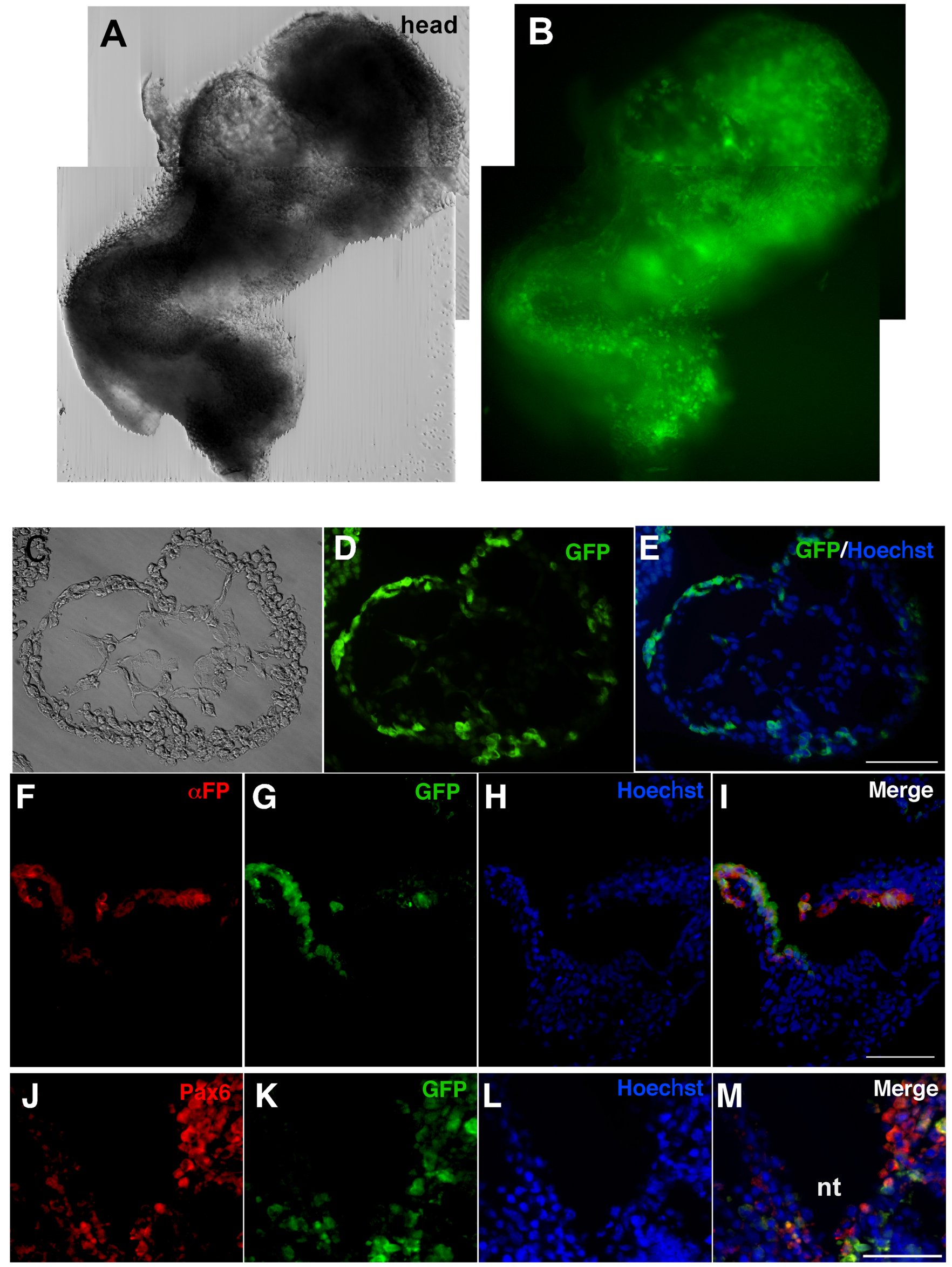


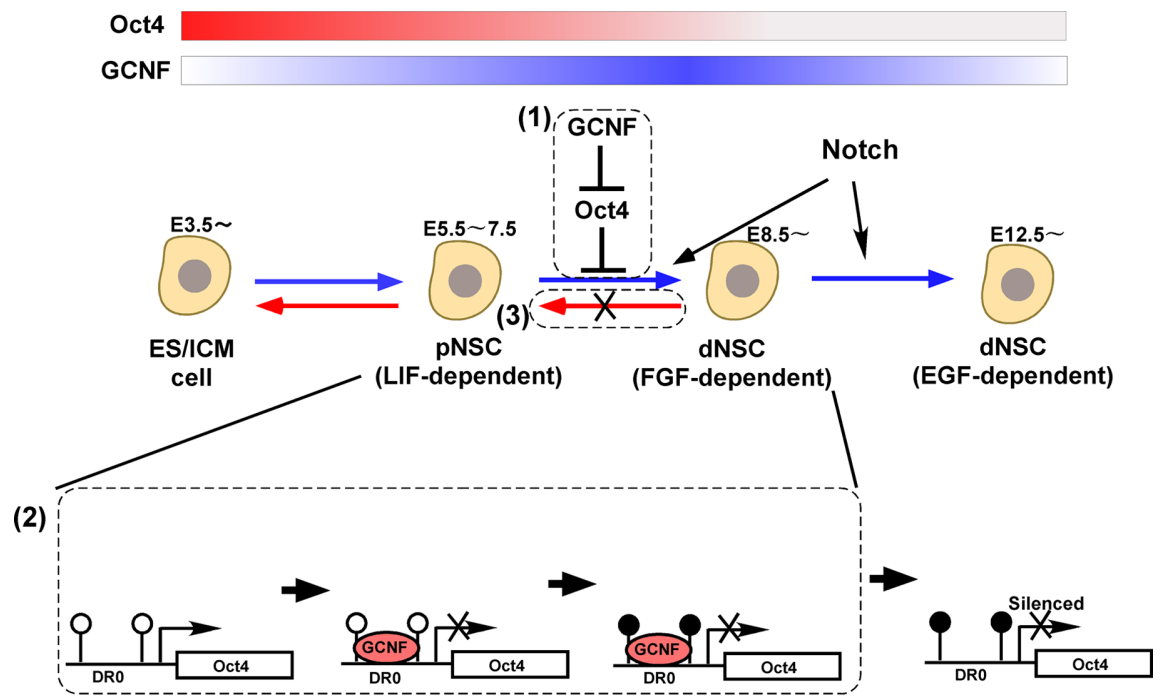

Figure 8. A model depicting the role of GCNF/Oct4 signaling in early NSC development. During neural differentiation from pluripotent ES or inner cell mass (ICM) cells, GCNF binds to the 0ct4 promoter in pNSCs and induces 0ct4 suppression that leads to the transition to dNSCs. GCNF induce de novo methylation of the 0ct4 promoter (Gu et al., 2006). Notch signaling also may be involved in the pNSC/dNSC transition, but more likely has an important role in the self-renewal of FGF-dependent dNSCs and their transition into EGF-dependent dNSCs. Oct4 remains suppressed by methylation even after GCNF is no longer expressed. Further details are mentioned in the text. DRO; Direct repeat element with 0 base pair spacing between the half sites.

tic effects of multiple pluripotent genes are required for the acquisition of pluripotency in somatic cells. Presumably, GCNF may epigenetically regulate these other three pluripotency factors as well. Therefore, we conclude that transition from pNSC to dNSC is regulated by Oct4 levels, but it is irreversible presumably due to the GCNF induced gene silencing of the Oct4 and other pluripotent genes, all of which are necessary for pluripotency, with none being sufficient individually.

As cells progress along the neural lineage from ES cells to pNSCs and to dNSCs, they lose pluripotency. Donor LIFdependent pNSCs, after injection into host blastocysts, can make some contribution to the resultant chimeric embryo, while dNSCs from mouse embryos and adults cannot (Tropepe et al., 2001; Seaberg et al., 2002; D’Amour and Gage, 2003). The present data suggest that both transcriptional and epigenetic suppression of the Oct4 gene by GCNF induce the loss of pluripotency in early NSCs. Indeed, some epigenetic changes must be required for the longer term loss of pluripotency in dNSCs from the later embryo and adult, because the expression of GCNF at the transition between pNSCs and dNSCs in vivo is transient. Decreased Oct4 also is responsible for the loss of pluripotency in trophoblast cells that are derived from the early morula (Niwa et al., 2005) and increased Oct 4 may be important for the acquisition of pluripotency in long-term cultured mesenchymal stem cells (Jiang et al., 2002). The recent observation that conditional Oct 4 knock-out using nestin/Cre (expressed between E9.5 and E 15.5) does not affect brain germinal zone neural progenitors (Lengner et al.,

\section{$\leftarrow$}

Figure 7. $\mathrm{GCNF}^{-1-} \mathrm{dNSC}$ have ability to contribute to non-neural tissue. $A, B$, Aggregated embryos were transplanted into host animals and analyzed at $E 9.0$. Bright field $(\boldsymbol{A})$ and fluorescent $(\boldsymbol{B})$ images of the same embryo show that $\mathrm{GCNF}^{-1-} \mathrm{dNSC}$ contributed broadly in the recovered embryos. $(-\boldsymbol{M}$, Sections of the heart $(\boldsymbol{C}-\boldsymbol{E})$, endoderm $(\boldsymbol{F}-\boldsymbol{I})$, and neural tube $(\boldsymbol{J}-\boldsymbol{M})$ immunostained with GFP $(\boldsymbol{D}, \boldsymbol{G}, \boldsymbol{K}), \operatorname{AFP}(\boldsymbol{F})$, and Pax6 $(\boldsymbol{J})$ or nuclear-stained by Hoechst 33342 $(\boldsymbol{H}, \boldsymbol{L})$ and merged images $(\boldsymbol{E}, \boldsymbol{I}, \boldsymbol{M})$. GFP-positive donor cells were found in the cardiac wall (D), $\alpha \mathrm{FP}$-positive endoderm $(\boldsymbol{I})$ and neural tube as Pax6 positive neural cells $(\boldsymbol{M})$. nt, Neural tube.
2007) is consistent with the present data showing that Oct4 is not necessary for the maintenance or proliferation of dNSCs.

Notch signaling also is important in early NSC development (Hitoshi et al., $2002,2004)$. However, upregulation of the Notch target gene Hes5 was observed in both $\mathrm{GCNF}^{+/+}$and $\mathrm{GCNF}^{-1-}$ cells during neural differentiation (supplemental Fig. S12B, available at www.jneurosci.org as supplemental material). Given that the numbers of dNSC-derived clonal neurospheres were decreased but not eliminated in Notch pathway mutants, Notch signaling may have an independent role in the transition from pNSCs to dNSCS. However, it seems more likely that Notch signaling does not prevent the transition from pNSC to dNSC, but simply inhibits the self renewal of the newly formed dNSCs, given that Notch pathway mutant dNSCs show a clearly compromised ability for selfrenewal (Hitoshi et al., 2002, 2004).

Changes in Oct4 levels in pluripotent ES cells clearly alter the fates of these cells. While increased Oct4 expression causes differentiation into primitive endoderm and mesoderm, repression of Oct4 induces loss of pluripotency and differentiation to trophectoderm (Niwa et al., 2000, 2005). In the neural lineage, experiments using tetracycline inducible Oct4 ES cells demonstrated that increased numbers of MAP2 positive neurons were produced from ES cells after sustained expression of Oct4 (Shimozaki et al., 2003). However, increased Oct4 does not appear to induce neural cell fate specification (Niwa et al., 2000,2005 ), and the current data suggest it positively inhibits the induction of dNSCs. We suggest that the reported increase in neurons after Oct4 overexpression in ES cells (Shimozaki et al., 2003) may be the result of a survival effect rather than a fate change. When ES cells are plated in the minimal media conditions that induce the differentiation into the neural lineage, $>70 \%$ of the newly formed neural cells die during this early neural differentiation from ES cells (Smukler et al., 2006). However, GCNF ${ }^{-1-}$ ES cells and pNSC, which have higher Oct4 expression, showed increased cell viability compared with WT cells. Thus, it is possible that the increased MAP2 positive cells seen after long-term differentiation in the presence of Oct4 overexpression (Shimozaki et al., 2003) was a by-product of the increased survival of the ES cells and early neural cells. Furthermore, the impaired neuronal differentiation of Oct4 downregulated ES cells (Niwa et al., 2000) can be explained more parsimoniously by a cell fate change from ES cells into the trophectoderm lineage.

In summary, we suggest novel roles for Oct4 and GCNF in vertebrate somatic cells (Fig. 8). (1) Oct4 suppression by GCNF is required for the transition from pNSC to dNSC which occurs between E7.5 and E8.5 in vivo. (2) GCNF also methylates the Oct4 promoter during this transition and maintains the long term silencing of the Oct4 gene in dNSCs. (3) Once pNSCs transition into dNSCs, the dNSCs lose their pluripotency and competency to dedifferentiate into pNSCs even if Oct4 is overexpressed, suggesting the epigenetic modification of other pluripotency genes by GCNF. 


\section{References}

Chambers I, Colby D, Robertson M, Nichols J, Lee S, Tweedie S, Smith A (2003) Functional expression cloning of Nanog, a pluripotency sustaining factor in embryonic stem cells. Cell 113:643-655.

Chung AC, Cooney AJ (2001) Germ cell nuclear factor. Int J Biochem Cell Biol 33:1141-1146.

Chung AC, Katz D, Pereira FA, Jackson KJ, DeMayo FJ, Cooney AJ, O’Malley BW (2001) Loss of orphan receptor germ cell nuclear factor function results in ectopic development of the tail bud and a novel posterior truncation. Mol Cell Biol 21:663-677.

Chung AC, Xu X, Niederreither KA, Cooney AJ (2006) Loss of orphan nuclear receptor GCNF function disrupts forebrain development and the establishment of the isthmic organizer. Dev Biol 293:13-24.

D'Amour KA, Gage FH (2003) Genetic and functional differences between multipotent neural and pluripotent embryonic stem cells. Proc Natl Acad Sci U S A 100 Suppl 1:11866-11872.

Fuhrmann G, Chung AC, Jackson KJ, Hummelke G, Baniahmad A, Sutter J, Sylvester I, Schöler HR, Cooney AJ (2001) Mouse germline restriction of Oct4 expression by germ cell nuclear factor. Dev Cell 1:377-387.

Gu P, LeMenuet D, Chung AC, Mancini M, Wheeler DA, Cooney AJ (2005) Orphan nuclear receptor GCNF is required for the repression of pluripotency genes during retinoic acid-induced embryonic stem cell differentiation. Mol Cell Biol 25:8507-8519.

Gu P, Le Menuet D, Chung AC, Cooney AJ (2006) Differential Recruitment of Methylated CpG Binding Domains by the Orphan Receptor GCNF Initiates the Repression and Silencing of Oct4 Expression. Mol Cell Biol 26:9471-9483.

Hitoshi S, Alexson T, Tropepe V, Donoviel D, Elia AJ, Nye JS, Conlon RA, Mak TW, Bernstein A, van der Kooy D (2002) Notch pathway molecules are essential for the maintenance, but not the generation, of mammalian neural stem cells. Genes Dev 16:846-858.

Hitoshi S, Seaberg RM, Koscik C, Alexson T, Kusunoki S, Kanazawa I, Tsuji S, van der Kooy D (2004) Primitive neural stem cells from the mammalian epiblast differentiate to definitive neural stem cells under the control of Notch signaling. Genes Dev 18:1806-1811.

Hummelke GC, Cooney AJ (2001) Germ cell nuclear factor is a transcriptional repressor essential for embryonic development. Front Biosci 6:D1186-1191.

Jiang Y, Jahagirdar BN, Reinhardt RL, Schwartz RE, Keene CD, OrtizGonzalez XR, Reyes M, Lenvik T, Lund T, Blackstad M, Du J, Aldrich S, Lisberg A, Low WC, Largaespada DA, Verfaillie CM (2002) Pluripotency of mesenchymal stem cells derived from adult marrow. Nature 418:41-49.

Karpowicz P, Inoue T, Runciman S, Deveale B, Seaberg R, Gertsenstein M, Byers L, Yamanaka Y, Tondat S, Slevin J, Hitoshi S, Rossant J, van der Kooy D (2007) Adhesion is prerequisite, but alone insufficient, to elicit stem cell pluripotency. J Neurosci 27:5437-5447.

Lan ZJ, Gu P, Xu X, Jackson KJ, DeMayo FJ, O’Malley BW, Cooney AJ (2003) GCNF-dependent repression of BMP-15 and GDF-9 mediates gamete regulation of female fertility. EMBO J 22:4070-4081.

Lengner CJ, Camargo FD, Hochedlinger K, Welstead GG, Zaidi S, Gokhale S, Scholer HR, Tomilin A, Jaenisch R (2007) Oct4 expression is not re- quired for mouse somatic stem cell self-renewal. Cell Stem Cell 1:403415.

Miyoshi H, Blömer U, Takahashi M, Gage FH, Verma IM (1998) Development of a self-inactivating lentivirus vector. J Virol 72:8150-8157.

Morshead CM, Reynolds BA, Craig CG, McBurney MW, Staines WA, Morassutti D, Weiss S, van der Kooy D (1994) Neural stem cells in the adult mammalian forebrain: a relatively quiescent subpopulation of subependymal cells. Neuron 13:1071-1082.

Nagy A GM, Vintersten K, Behringer R (2002) Manipulating the mouse embryo: a laboratory manual. Cold Spring Harbor, NY: Cold Spring Harbor Laboratory.

Nichols J, Zevnik B, Anastassiadis K, Niwa H, Klewe-Nebenius D, Chambers I, Schöler H, Smith A (1998) Formation of pluripotent stem cells in the mammalian embryo depends on the POU transcription factor Oct4. Cell 95:379-391.

Niwa H, Miyazaki J, Smith AG (2000) Quantitative expression of Oct-3/4 defines differentiation, dedifferentiation or self-renewal of ES cells. Nat Genet 24:372-376.

Niwa H, Toyooka Y, Shimosato D, Strumpf D, Takahashi K, Yagi R, Rossant J (2005) Interaction between Oct3/4 and Cdx2 Determines Trophectoderm Differentiation. Cell 123:917-929.

Orkin SH (2005) Chipping away at the embryonic stem cell network. Cell $122: 828-830$.

Reynolds BA, Weiss S (1992) Generation of neurons and astrocytes from isolated cells of the adult mammalian central nervous system. Science 255:1707-1710.

Seaberg RM, Hitoshi S, Tropepe V, Karpowicz P, Cheah YC, Rossant J, van der Kooy D (2002) The identification and chimeric characterization of primitive and definitive mammalian neural stem cells. Soc Neurosci Abstr 28:726.1.

Shimozaki K, Nakashima K, Niwa H, Taga T (2003) Involvement of Oct3/4 in the enhancement of neuronal differentiation of ES cells in neurogenesis-inducing cultures. Development 130:2505-2512.

Smith A (2005) The Battlefield of Pluripotency. Cell 123:757-760.

Smukler SR, Runciman SB, Xu S, van der Kooy D (2006) Embryonic stem cells assume a primitive neural stem cell fate in the absence of extrinsic influences. J Cell Biol 172:79-90.

Süsens U, Aguiluz JB, Evans RM, Borgmeyer U (1997) The germ cell nuclear factor mGCNF is expressed in the developing nervous system. Dev Neurosci 19:410-420.

Tahara-Hanaoka S, Sudo K, Ema H, Miyoshi H, Nakauchi H (2002) Lentiviral vector-mediated transduction of murine CD34(-) hematopoietic stem cells. Exp Hematol 30:11-17.

Takahashi K, Yamanaka S (2006) Induction of pluripotent stem cells from mouse embryonic and adult fibroblast cultures by defined factors. Cell 126:663-676

Tropepe V, Hitoshi S, Sirard C, Mak TW, Rossant J, van der Kooy D (2001) Direct neural fate specification from embryonic stem cells: a primitive mammalian neural stem cell stage acquired through a default mechanism. Neuron 30:65-78.

Yeom YI, Fuhrmann G, Ovitt CE, Brehm A, Ohbo K, Gross M, Hübner K, Schöler HR (1996) Germline regulatory element of Oct-4 specific for the totipotent cycle of embryonal cells. Development 122:881-894. 